\title{
Evaluation of ibuprofen loaded solid lipid nanoparticles and its combination regimens for pancreatic cancer chemoprevention
}

\author{
ARVIND THAKKAR * , SUSHMA CHENREDDY* ${ }^{*}$ JEFFREY WANG and SUNIL PRABHU \\ Department of Pharmaceutical Sciences, College of Pharmacy, \\ Western University of Health Sciences, Pomona, CA 91766, USA
}

Received December 2, 2014; Accepted January 5, 2015

DOI: $10.3892 /$ ijo.2015.2879

\begin{abstract}
The objective of the present study was to establish the individual and combined chemopreventive potential of a widely used non-steroidal anti-inflammatory drug, ibuprofen (IBU), encapsulated in solid lipid nanoparticles (SLNs) for the chemoprevention of pancreatic cancer. The IBU SLNs were optimized using various lipids (Stearic acid, Compritol 888 ATO and Tripalmitin) and surfactants (Poloxamer 188, Tween-80). The synergistic effect of combination of IBU with sulforaphane (SFN) was also evaluated. Cell viability studies were conducted followed by colony formation and $\mathrm{NF}-\kappa \mathrm{B}$ DNA binding assays. The $\mathrm{IC}_{50}$ concentration of free IBU in human pancreatic cancer Panc-1 and MIA PaCa-2 cells were 1.25 and $1.26 \mathrm{mM}$, respectively. SLN optimization study of IBU revealed stearic acid (1:2 drug to lipid ratio) formulated with Poloxamer 188 to be the most efficacious in cell viability study. Upon encapsulation in SLNs, $\mathrm{IC}_{50}$ concentration of IBU-SLN was 113.8 and $122.6 \mu \mathrm{M}$ for Panc-1 and MIA PACa-2 cells, respectively, reflecting a 10 -fold reduction compared to free IBU. Combinations of low doses of free IBU $(250 \mu \mathrm{M})$ and SFN $(5 \mu \mathrm{M})$ reduced cell viability by $\sim 55 \%(\mathrm{P}<0.01)$, whereas a lower dose of encapsulated IBU-SLN $(62.5 \mu \mathrm{M})$ and free SFN $(5 \mu \mathrm{M})$ reduced cell viability by $\sim 80 \%$ ( $\mathrm{P}<0.001)$ for both Panc-1 and MIA PaCa-2 cells. These results reflect 4 -fold reduction in IBU-SLN dose in combination compared to free IBU. Moreover, IBU-SLN and free SFN combination reduced number of colonies by $\sim 50 \%$ ( $\mathrm{P}<0.01)$. Further, IBU-SLN and SFN combinations showed down-regulation of DNA binding activity of the p50
\end{abstract}

Correspondence to: Dr Sunil Prabhu, Department of Pharmaceutical Sciences, College of Pharmacy, Western University of Health Sciences, 309 East 2nd Street, Pomona, CA 91766, USA

E-mail: sprabhu@westernu.edu

${ }^{*}$ Contributed equally

Key words: chemoprevention, pancreatic cancer, solid lipid nanoparticles, ibuprofen, sulforaphane, drug delivery system subunit of NF- $\kappa \mathrm{B}$. In conclusion, these preliminary results demonstrate the potential of IBU as a chemopreventive agent against pancreatic cancer. Furthermore, when encapsulated in nanotechnology-based SLN delivery systems and delivered in combination with SFN provide evidence of a promising approach for pancreatic cancer prevention and therapy.

\section{Introduction}

Pancreatic cancer is the fourth most common cause of cancer deaths in the US with a five-year survival rate of $<5 \%$. According to the American Cancer Society, in the US alone it is estimated that 46,420 individuals will be diagnosed with and 39,590 of them will die of pancreatic cancer in 2014 (1). The low survival rate of patients points towards an increased need for novel strategies to combat this deadly disease. The concept of chemoprevention has recently received significant attention as a novel strategy for pancreatic cancer $(2,3)$. Moreover, use of a combination of chemopreventive agents that differ in their mode of action and target multiple pathways has garnered recent attention $(4,5)$. This approach provides a means of low-dose therapy with increased efficacy and less toxicity. However, combination therapy studies specifically for pancreatic cancer prevention are still in its infancy.

Numerous epidemiological and animal studies have suggested that commonly used non-steroidal anti-inflammatory drugs (NSAIDs) such as aspirin can reduce incidence and mortality of many types of cancer $(2,3)$. More recently, ibuprofen (IBU), also an NSAID, have been reported to inhibit promotion and proliferation of tumors in in vitro and in vivo studies (6-8). Although promising in its chemopreventive potential, IBU's adverse effects such as increased gastrointestinal ulceration may prevent long-term use $(9,10)$. Encapsulation within nanoparticle formulations may offer the opportunity to reduce side effects of these drugs while maintaining high efficacy as shown in recent literature $(11,12)$. Lipid nanoparticles with a solid matrix, such as solid lipid nanoparticles (SLNs) and polymeric nanoparticles are examples of formulations which may be useful in chemoprevention $(2,3,13)$. Nanosized drug delivery systems such as SLNs offer several advantages over conventional delivery system including controlled and sustained release of drugs, ability of the drug to cross the 
mucosal barriers, decreased renal and hepatic clearance, decreased immune recognition, increased apparent halflives of drugs, and increased stability and solubility $(14,15)$. However, the most important advantage of SLNs is that they can increase the oral bioavailability of lipophilic drugs. The emerging role of nanoparticles in cancer therapy and chemoprevention justifies a need for further research in this area.

Sulforaphane (SFN) is a naturally occurring sulfurcontaining isothiocyanate found in cruciferous vegetables such as broccoli, Brussel's sprouts, cauliflower, and cabbage (16). SFN has been shown to be not only effective in preventing various chemically induced cancers in animal models, but also inhibits the growth of established tumors (17-19). SFN has been shown to reduce NF- $\kappa B$ activity and affect expression of NF- $\kappa B$ mediated genes encoding adhesion molecules, inflammatory cytokines, growth factors, and anti-apoptotic factors (20).

In this study, IBU-loaded SLN formulations were optimized by using i) stearic acid, ii) compritol ATO 888 and iii) tripalmitin as the lipid matrices mixed with either a) Poloxamer 188 or b) Tween-80 as the surfactant. Particle size, entrapment efficiency, zeta potential and in vitro drug dissolution rates of the resulting IBU-SLNs were investigated. To date, no other group has investigated the effects of low-dose free IBU, IBU-SLNs or IBU-SLN combined with free SFN on pancreatic cancer cells. Thus, we optimized IBU-SLN formulations to evaluate their combined chemopreventive efficacy in Panc-1 and MIA $\mathrm{PaCa}-2$ human pancreatic cancer cells.

\section{Materials and methods}

Reagents. IBU was obtained from LKT Laboratories (St. Paul, MN, USA). SFN was obtained from Santa Cruz Biotechnology (Santa Cruz, CA, USA). Stearic acid was obtained from JT Baker (Center Valley, PA, USA), tripalmitin from Tokyo Chemical Industry (Tokyo, Japan) and compritol ATO 888 from Gattefossse (France). Poloxamer 188 and Tween- 80 was obtained from Spectrum Chemicals (Gardena, CA, USA). HPLC grade acetonitrile was purchased from BDH (Radnor, PA, USA), and ortho-phosphoric acid from Fisher Scientific (Fair Lawn, NJ, USA).

Human pancreatic cancer cell lines. Panc-1 and MIA PaCa-2 cell lines were obtained from ATCC (Rockville, MD, USA). Cells were maintained in Dulbecco's modified Eagle's medium (DMEM) containing 10\% fetal bovine serum obtained from ATCC. Cells were cultured at $37^{\circ} \mathrm{C}$ in a humidified atmosphere of $5 \% \mathrm{CO}_{2}$ and $95 \%$ air.

Preparation of solid lipid nanoparticles. Ibuprofen SLNs were prepared using a hot melt oil-in-water $(\mathrm{o} / \mathrm{w})$ emulsion technique. Stearic acid, compritol and tripalmitin lipids were used separately to optimize the nanoparticle formulations. Briefly, $400 \mathrm{mg}$ of each lipid was melted at $70^{\circ} \mathrm{C}$. IBU $(200 \mathrm{mg})$ was dissolved in the cooling melted lipid. The water phase consisted of $2 \%$ Poloxamer or $2 \%$ Tween- 80 which was heated to the same temperature as that of the lipid phase. The lipid phase was then added drop wise to the surfactant solution using continuous high sheer homogenization. Thereafter, the mixture was sonicated for $1 \mathrm{~min}$ using a probe-sonicator (Branson, Pomona, CA, USA) to form an emulsion. The emulsion was cooled and the resulting IBU-SLNs were freeze-dried in a freeze dryer (Labconco, Kansas City, MO, USA) and subjected to particle size, encapsulation efficiency and zetapotential determination.

Determination of mean particle size, polydispersity index and zeta potential. The mean particle size (z-average) and polydispersity index (PDI) as a measure of the width of particle size distribution is determined by photon correlation spectroscopy using Zetasizer (Nano ZS 90, Malvern Instruments, Malvern, UK) at $25^{\circ} \mathrm{C}$ and $90^{\circ}$ scattering angle. SLN formulation was diluted with nano-pure water to weaken opalescence before measurements. The surface charge was assessed by measuring zeta potential of SLNs based on the Smoluchowski Equation, using the same equipment at $25^{\circ} \mathrm{C}$ with electric field strength of $23 \mathrm{~V} / \mathrm{cm}(21)$.

Determination of \% encapsulation efficiency of IBU-SLNs. Encapsulation efficiency was determined by dissolving $10 \mathrm{mg}$ of the SLN formulation in $10 \mathrm{ml}$ acetonitrile solvent. The drug was released from the lipid into acetonitrile and allowed to dissolve freely for $10 \mathrm{~min}$ in a sonicator after which it was filtered through a $0.45-\mu \mathrm{m}$ filter. The resulting solution was further diluted with acetonitrile and was analyzed by using a Shimadzu LC-20 binary HPLC system (Columbia, MD, USA). Caffeine was used as the internal standard.

The entrapment efficiency was calculated using the following formula: EE $(\%)=$ Amount $(\mathrm{mg})$ of drug per HPLC method/theoretical yield (mg) x100.

In vitro drug release from IBU-SLNs. The cumulative release of IBU from SLNs was determined in phosphate buffered saline (PBS), pH 7.4. SLNs containing $5 \mathrm{mg}$ of the IBU-SLNs was suspended in $50 \mathrm{ml}$ of PBS and placed in an incubator at $37^{\circ} \mathrm{C}$ with a shaking speed of $100 \mathrm{rpm}$. Drug release from SLN was compared to PBS with blank SLNs. At predetermined time intervals $(0,0.5,1,2,4,6,12,24,48,72$ and $96 \mathrm{~h}), 1 \mathrm{ml}$ of the buffer was withdrawn and replaced with equivalent volume of fresh buffer. All samples are centrifuged at $5000 \mathrm{rpm}$ for $10 \mathrm{~min}$. The amount of released drug was analyzed using HPLC. The analysis was carried out in triplicate.

Chromatographic analysis of IBU-SLNs. IBU was analyzed using a Shimadzu LC-20 binary HPLC system. The system consists of a Restek Ultra II C-18 column (4.6x150 mm, $5 \mu \mathrm{m})$ with mobile phase composed of acetonitrile and $1 \%$ orthophosphoric acid (60:40). The flow rate was set at $0.5 \mathrm{ml} / \mathrm{min}$ and the detection was at $220 \mathrm{~nm}$ using a photo diode array detector. The retention time of IBU was $10.7 \mathrm{~min}$.

Cell viability assay. The cell viability assay was performed according to manufacturer's protocol with the Promega CellTitre 96 Aqueous MTS reagent (Madison, WI, USA). Briefly, $7.5 \times 10^{3}$ cells were seeded in 96 -well plates and incubated overnight. The test compounds IBU and SFN were added and incubated for a period of $72 \mathrm{~h}$. At the end of the incubation period, the growth medium was removed followed by addition of $100 \mu \mathrm{l}$ media consisting of $20 \%$ MTS and $1 \%$ of phenazine methosulfate (PMS) and the mixture was incubated for $2 \mathrm{~h}$ at $37^{\circ} \mathrm{C}$. MTS is bio-reduced by the cells into formazan which 
Table I. Optimization of ibuprofen solid lipid nanoparticle (IBU-SLN) formulations.

\begin{tabular}{lcccccc}
\hline Lipid & $\begin{array}{c}\text { Surfactant } \\
(2 \%)\end{array}$ & $\begin{array}{c}\text { Drug:Lipid } \\
\text { ratio }\end{array}$ & $\begin{array}{c}\text { Particle } \\
\text { size }(\mathrm{nm})\end{array}$ & $\begin{array}{c}\text { Encapsulation } \\
\text { efficiency }(\% \mathrm{EE})\end{array}$ & $\begin{array}{c}\text { Zeta potential } \\
(\mathrm{mV})\end{array}$ & $\begin{array}{c}\text { Polydispersity } \\
\text { index (PDI) }\end{array}$ \\
\hline Stearic acid & Tween-80 & $1: 2$ & 170 & 63 & -13.7 & 0.3 \\
& & $1: 4$ & 450 & 66.4 & -15.8 & 0.4 \\
& Poloxamer 188 & $1: 2$ & 485 & 69 & -13.2 & 0.3 \\
& & $1: 4$ & 650 & 71 & -7.11 & 0.2 \\
Compritol 888 ATO & Tween-80 & $1: 2$ & 291 & 64 & -0.8 & 0.4 \\
& & $1: 4$ & 350 & 69.4 & -3.76 & 0.2 \\
& Poloxamer 188 & $1: 2$ & 543 & 70.2 & -13.2 & 0.2 \\
Tripalmitin & & $1: 4$ & 595 & 87 & -2.53 & 0.4 \\
& Tween-80 & $1: 2$ & 207 & 66 & -11.5 & 0.4 \\
& & $1: 4$ & 335 & 68 & -14.3 & 0.3 \\
& \multirow{2}{*}{ Poloxamer 188 } & $1: 2$ & 390 & 66 & -18.4 & 0.2 \\
\hline
\end{tabular}

could be measured at $490 \mathrm{~nm}$. Thus, the quantity of formazan product measured by absorbance is directly proportional to the number of living cells in culture. $\mathrm{IC}_{50}$ values were determined using GraphPad Prism software (San Diego, CA, USA). All analysis was performed in triplicate. Each experiment was repeated at least once.

Colony formation assay. Cells $\left(1 \times 10^{4}\right)$ were seeded into 24-well plates in triplicate per data point. Cells were treated with blank-SLN, IBU-SLN, SFN alone and in combinations IBU-SLN+SFN. Two weeks after treatment, cells were fixed and stained with $0.5 \%$ crystal violet (Sigma, St. Louis, MO, USA) in methanol for $5 \mathrm{~min}$. Colonies consisting of 50 or more cells were counted. The percentage cell survival was calculated (Plating efficiency of non-treated cultures $=1$ ).

$N F-\kappa B$ DNA binding assay. The DNA-binding activity of NF- $\kappa \mathrm{B}$ was quantified by ELISA, using the TransAM NF- $\kappa \mathrm{B}$ p50 transcription factor assay kit (Active Motif, Carlsbad, CA, USA). Briefly, $30 \mu \mathrm{g}$ of total protein was incubated in a 96-well plates coated with immobilized oligonucleotide for the p50 subunit. NF- $\kappa \mathrm{B}$ binding to the target oligonucleotide was detected by incubation with primary antibody specific for the activated form of p50 (active motif), visualized and quantified at $490 \mathrm{~nm}$.

Statistical analysis. GraphPad Prism software was used for statistical analysis and graph plotting. The results are expressed as mean \pm SEM and analyzed by one-way ANOVA followed by Tukey's post-hoc test. The $\mathrm{IC}_{50}$ values were calculated using nonlinear regression and plotted as log (inhibitor) vs. response (variable slope) curve. A probability value of $\leq 0.05$ was considered significant.

\section{Results}

Effect of different lipid materials on particle size of IBU-SLNs. A summary of all formulations with their respective preparation conditions and characterization is presented in Table I. In order to optimize IBU-SLN formulations, different lipid materials (stearic acid, Compritol and tripalmitin) were used. In addition, IBU-SLNs were prepared with drug to lipid ratio of 1:2 or $1: 4$, and either with $2 \%$ Poloxamer or $2 \%$ Tween- 80 in the aqueous phase. In the tested parameters range, we observed that the mean particle diameter (z-average) of IBU-SLNs increased with an increase in lipid concentration from 1:2 (170-543 nm) to 1:4 (335-650 nm). Thus, increase in lipid concentrations resulted in larger particle size. With respect to the use of surfactant, Tween-80 based IBU-SLNs showed smaller particle size $(170-450 \mathrm{~nm})$ compared to Poloxamer 188 (390-650 nm) based SLN formulations.

Effect of various formulation parameters on encapsulation efficiency (\% EE) of IBU-SLNs. As shown in Table I, the amount of lipid has significant effect on the encapsulation efficiency. There was an increase in $\%$ EE from drug to lipid ratio of 1:2 (63-70.2\%) to 1:4 (66.4-87\%). Specifically, Poloxamer containing SLN formulations showed an increase in \% EE (66-87\%) compared to Tween-80 (63-69.4\%) based SLN formulations.

Effect of ratio of drug to lipid on zeta potential of IBU-SLNs. As shown in Table I, the change of surfactant significantly affected the zeta potential of SLNs. In the case of Poloxamer 188, the zeta-potential charge on SLNs were reduced significantly with drug to lipid ratio of $1: 2(-13.2$ to $-18.4 \mathrm{mV})$ to $1: 4(-2.53$ to $-7.11 \mathrm{mV})$, whereas there was increase in zeta potential in Tween-80 formulation with drug to lipid ratio from $1: 2(-0.8$ to $-13.7 \mathrm{mV})$ to $1: 4(-3.76$ to $-15.8 \mathrm{mV})$.

Determination of cell viability of free IBU on pancreatic cancer cells. In order to evaluate the effect of free IBU on pancreatic cancer, the dilution range of IBU (5-5000 $\mu \mathrm{M})$ were added to Panc- 1 and MIA PaCa-2 cells for $72 \mathrm{~h}$. Our observations indicated a dose-dependent reduction in cell viability 
Table II. The $\mathrm{IC}_{50}$ values of free IBU and IBU-SLNs.

\begin{tabular}{lcccc}
\hline & Formulations & & & \\
\hline Drug & Surfactant & $\begin{array}{c}\text { Drug:lipid } \\
\text { ratio }\end{array}$ & $\begin{array}{c}\text { Panc-1 cells } \\
\left(\mathrm{IC}_{50} \text { values } \mu \mathrm{M}\right)\end{array}$ & $\begin{array}{c}\text { MIA PaCa-2 cells } \\
\left(\mathrm{IC}_{50} \text { values } \mu \mathrm{M}\right)\end{array}$ \\
\hline Free-Ibuprofen & & & 1245 & 1263 \\
IBU-stearic acid SLNs & Poloxamer 188 & $1: 2$ & 113.8 & 122.6 \\
& & $1: 4$ & 399.3 & 348.0 \\
IBU-compritol SLNs & Poloxamer 188 & $1: 2$ & 408.0 & 470.4 \\
& & $1: 4$ & 539.5 & 531.4 \\
IBU-tripalmitin SLNs & Poloxamer 188 & $1: 2$ & 586.0 & 554.3 \\
& & $1: 4$ & 639.3 & 631.7 \\
\hline
\end{tabular}

A

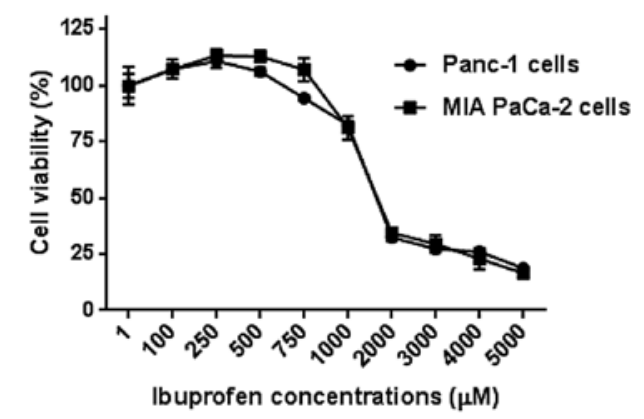

B

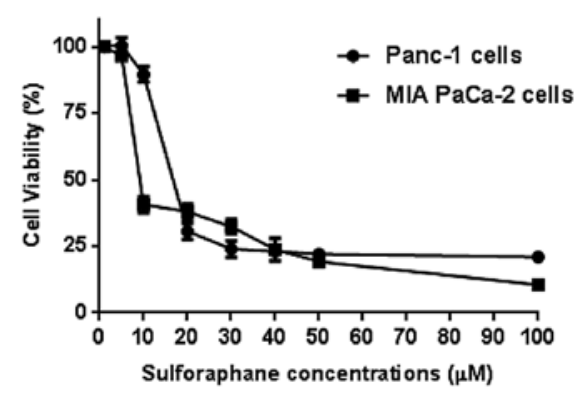

Figure 1. Dose-dependent inhibition of cell viability in pancreatic cancer cells. MIA PaCa-2 and Panc-1 cells were treated with (A) Ibuprofen (IBU) and (B) Sulforaphane (SFN). MTS assay was performed to determine the cell viability of Panc-1 and MIA PaCa-2 cells after treating with a range of concentrations of free IBU for $72 \mathrm{~h}$. The $\mathrm{IC}_{50}$ values were then determined using nonlinear regression using graph pad prism software. Each data point represents the mean percent viable cells measured in three parallel but independent experiments.

with $\mathrm{IC}_{50}$ concentrations of 1.25 and $1.26 \mathrm{mM}$ in Panc- 1 and MIA PaCa-2 cells, respectively (Table II and Fig. 1).

Effect of IBU-SLN formulation parameters on cell viability of pancreatic cancer cells. To verify the effect of IBU-SLNs, cell viability assay of various formulations were carried out on pancreatic cancer cells. As shown in Fig. 2A, IBU-SLN formulations with Poloxamer as the surfactant and a 1:2 drug to lipid ratio showed that stearic acid based IBU-SLNs demonstrate maximal effect, inhibiting cell proliferation by $\sim 75 \%$ compared to blank-SLNs $(\mathrm{P}<0.001)$. This formulation showed the lowest $\mathrm{IC}_{50}$ of 113.8 and $122.6 \mu \mathrm{M}$ in Panc-1 and MIA $\mathrm{PaCa}-2$ cells, respectively. The most striking result emerging from this study was that the $\mathrm{IC}_{50}$ values of IBU-SLNs were at least 10 -fold lower than that of free IBU. The Compritol and tripalmitin based SLN formulation showed $20 \%$ decrease in cell viability of pancreatic cancer cells, with $\mathrm{IC}_{50}$ values in the range of 408.0-586.0 $\mu \mathrm{M}$.

The data in Fig. 2B represents cell viability study with Poloxamer as the surfactant and a 1:4 drug to lipid ratio. Stearic acid based IBU-SLNs inhibited cell viability by $\sim 45 \%$ in pancreatic cancer cells $(\mathrm{P}<0.01)$. Further analysis showed $\mathrm{IC}_{50}$ values of 399.3 and $348.0 \mu \mathrm{M}$ in Panc-1 and MIA PaCa-2 cells, respectively. Comparing the two results in Fig. 2A and B, it can be seen that the IBU-SLN formulation with stearic acid as the lipid, Poloxamer as the surfactant and a 1:2 drug to lipid ratio showed significant reduction in cell proliferation thus indicating maximum efficacy; subsequently, these formulations were selected for further studies.

Effect of the combination of IBU-SLN with SFN on pancreatic cancer cells. To examine the effect of combined regimen on cell proliferation, Panc- 1 and MIA PaCa- 2 cells were treated with low and ineffective concentrations of free-IBU $(250 \mu \mathrm{M})$ in combination with SFN $(5 \mu \mathrm{M})$ for $72 \mathrm{~h}$. As shown in Fig. 3A, single agents did not show significant change in cell viability at these concentrations. However, when used in combination at identical concentrations, IBU+SFN showed a significant effect with a reduction in cell viability of $\sim 55 \%(\mathrm{P}<0.01)$ in Panc-1 and MIA PaCa-2 cells, respectively.

After determining the optimal formulation conditions and dose response curves individually, IBU-SLNs $(62.5 \mu \mathrm{M})$ and free SFN $(5 \mu \mathrm{M})$ were selected showing minimal inhibitory response on the cell lines when used individually. However, when IBU SLNs+SFN combinations were used at the same concentrations, the cell viability was reduced by $\sim 80 \%$ for Panc-1 and MIA PaCa-2 cells, respectively ( $\mathrm{P}<0.001$; Fig. 3B). Thus, the combination of IBU-SLNs and SFN showed 4-fold lower concentration as compared to free IBU in the reduction of cell viability of pancreatic cancer cells. 
A

Poloxamer (1:2)

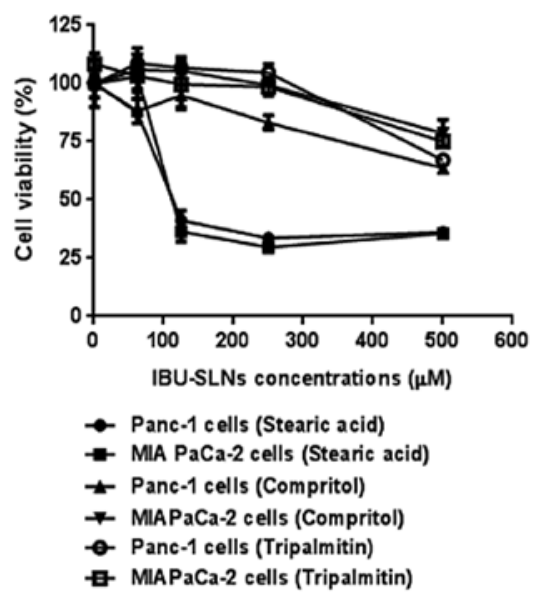

B

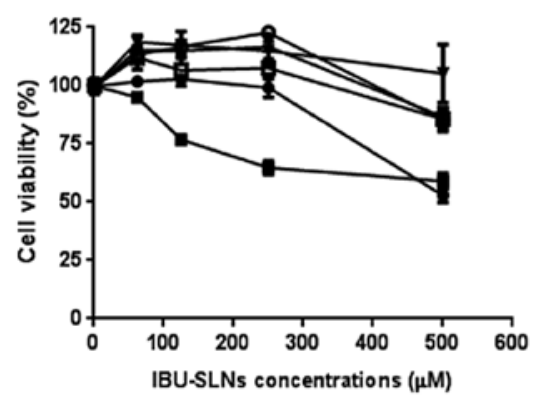

Figure 2. Effect of formulation parameters on ibuprofen (IBU) solid lipid nanoparticles (SLNs) on pancreatic cancer cell viability. IBU-SLN formulation with (A) Poloxamer as the surfactant and a 1:2 drug to lipid ratio; (B) Poloxamer as the surfactant and a 1:4 drug to lipid ratio. Each data point represents the mean percent viable cells measured in three parallel but independent experiments. The $\mathrm{IC}_{50}$ values were then determined using graph pad prism software.

In vitro evaluation of IBU drug release from IBU-SLNS. The in vitro drug release curve for the IBU-SLN suspension in $\mathrm{pH} 7.4$ phosphate buffer at $37^{\circ} \mathrm{C}$ is shown in Fig. 4. The drug release exhibited biphasic pattern, a burst drug release followed by sustained drug release. The IBU release was $40 \%$ during the first $2 \mathrm{~h}$; subsequently, sustained release of IBU from optimized IBU-SLNs was observed for the next $90 \mathrm{~h}$ release period.

Cell colony formation assay of IBU-SLNs. To evaluate longterm efficacy of IBU-SLNs on cell survival, a cell colony assay was performed. The survival fraction of the control group (blank-SLN) was set at 1 (representing 100\% cell survival) and the cell survival fraction was calculated based on individual and combination treatment. An evaluation of cell survival on MIA PaCa-2 cells showed survival fractions of 0.82 (IBU), and 0.89 (SFN), whereas IBU-SLN+SFN combination showed significant decrease in the survival fraction of $0.48(\mathrm{P}<0.01)$ (Fig. 5A). Similar results were observed in the case of Panc-1 with low survival fractions of cells.

Effect of IBU-SLNs in combination with SFN on DNA binding activity of $N F-\kappa B$. To gain further insight into the mechanism associated with the combination IBU-SLN and SFN, the DNA
A

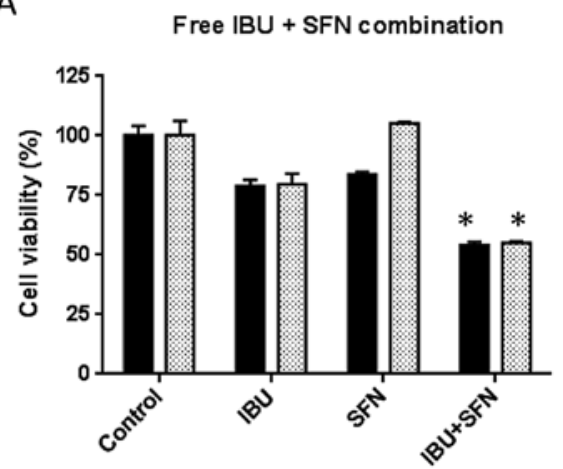

B

IBU SLN + SFN combination

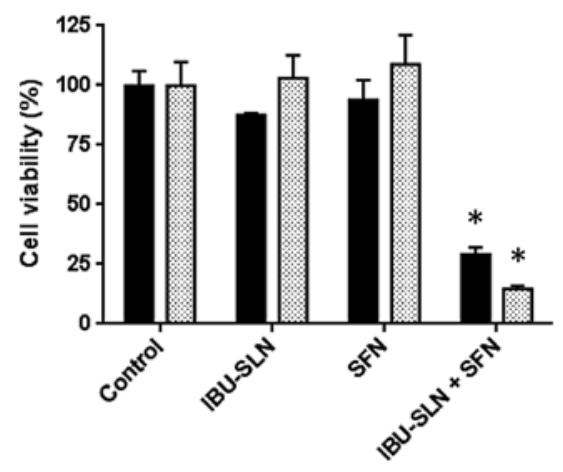

Panc-1 cells

MIA PaCa-2 cells

Figure 3. Synergistic effect of ibuprofen (IBU) and sulforaphane (SFN) combination on cell viability. MTS assay was performed to determine the cell viability of Panc-1 and MIA PaCa-2 cells. (A) Cells were treated with low dose of free ibuprofen (IBU; $250 \mu \mathrm{M}$ ) and sulforaphane (SFN; $5 \mu \mathrm{M}$ ) individually and in combination of IBU+SFN for $72 \mathrm{~h}$. (B) Cells were treated with IBU-SLNs $(62.5 \mu \mathrm{M})$, and free SFN $(5 \mu \mathrm{M})$ individually and in combination of IBU-SLN+SFN for $72 \mathrm{~h}$. Each bar represents the mean percent viable cells measured in three parallel but independent experiments. Statistical significance was determined by one-way ANOVA followed by Tukey's post hoc analysis. " $\mathrm{P}<0.05$ represents statistical significance of difference between control and treatment groups.

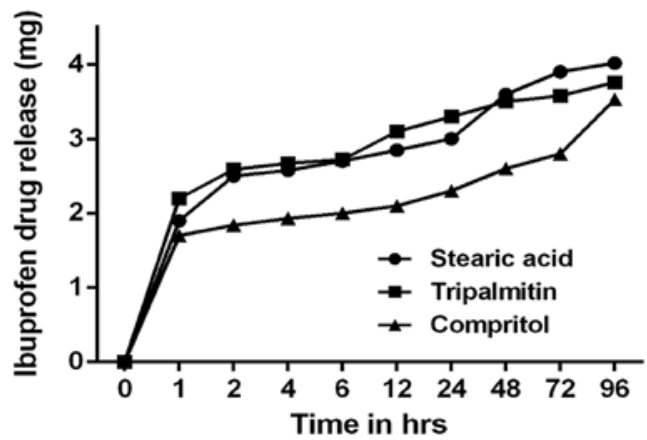

Figure 4. In vitro ibuprofen (IBU) release from IBU-SLNs over a period of $96 \mathrm{~h}$. The drug release was analyzed using an HPLC system. The data are plotted as mean \pm SEM. All the samples were analyzed in triplicate.

binding activity of the $\mathrm{p} 50$ subunit of the $\mathrm{NF}-\kappa \mathrm{B}$ complex was evaluated. As shown in Fig. 5B, 35\% decrease was observed in Panc-1 cells with the stearic acid based formulation, whereas MIA PaCa-2 cells showed $\sim 30 \%(\mathrm{P}<0.05)$ reduction in DNA binding activity of NF- $\mathrm{B}$ compared to blank SLN treatment group. 

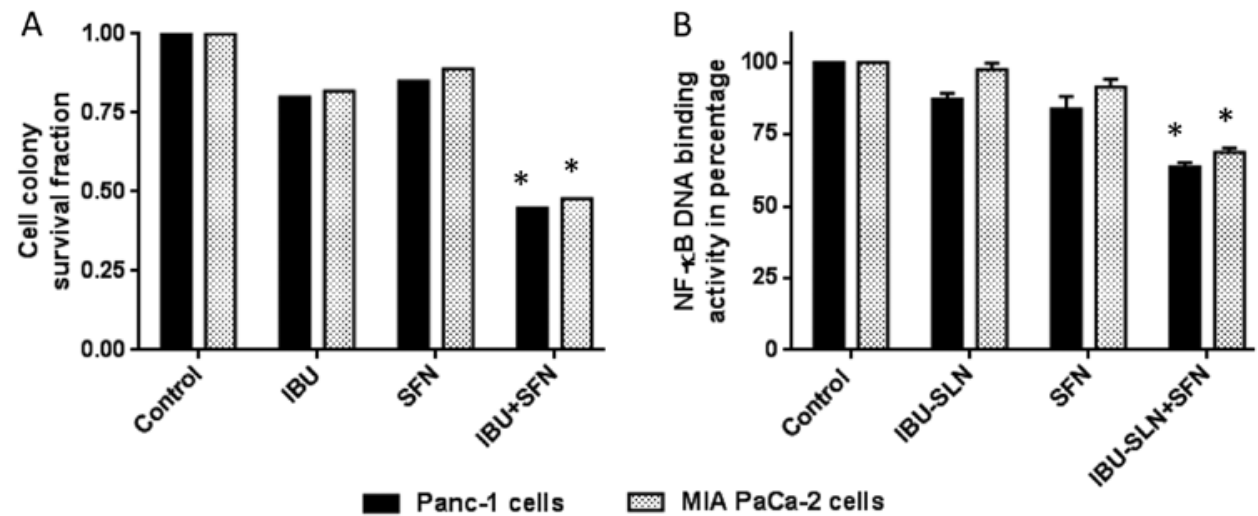

Figure 5. Combined effect of ibuprofen (IBU) and sulforaphane (SFN) on cell colony assay and NF- $\mathrm{B}$ activiation. (A) Cell colony formation assay showing survival fraction of IBU-solid lipid nanoparticles (SLNs) and SFN individually and in combination of IBU-SLN+SFN in Panc-1 and MIA PACa-2 pancreatic cancer cells. (B) Panc-1 and MIA PACa-2 cells were treated with IBU-SLN, and SFN individually or in combination for $24 \mathrm{~h}$. Total protein was extracted and incubated in 96-well plate coated with DNA binding site for the p50 subunit of NF- $\mathrm{KB}$. Statistical significance was determined by one-way ANOVA followed by Tukey's post hoc analysis. "P $<0.05$ represents statistical significance of difference between control and treatment groups.

\section{Discussion}

Early diagnosis of pancreatic cancer is difficult because it develops without any early symptoms. Low survival rate of patients with pancreatic cancer makes this disease of great concern (1). Millions of people routinely take aspirin, ibuprofen and other NSAIDs for control of pain and inflammation, or prophylaxis against cardiovascular disease. The widespread use of these compounds has facilitated epidemiological studies about their effect against cancer development. Numerous epidemiological, clinical, and laboratory studies have suggested that IBU, a commonly used NSAID, inhibits the proliferation of various tumors (6-8). Bonelli et al demonstrated the anti-proliferative effects of IBU on the human gastric cancer cell MKN-45. Free IBU, at concentration of $400-800 \mu \mathrm{M}$, could not inhibit cellular proliferation in a timeand dose-dependent manner, but $200 \mu \mathrm{M}$ IBU-PLGA could significantly inhibit the growth of gastric cancer MKN-45 cells (8). It is important to note that cancer preventive doses should be well below the therapeutic dose for the treatment of inflammatory conditions to avoid long-term toxicity. Hence, part of our current research also focused on the administration of low doses of IBU-SLNs to study its chemopreventive effects against Panc-1 and MIA PaCa-2 pancreatic cancer cells.

Appropriate choice of lipids, surfactants, and their composition affects the particle size of IBU-SLNs, drug encapsulation efficiency, and drug release behavior. Optimal SLN formulation for each drug that can be obtained by investigating the effect of process variables on the characteristics of the SLNs (22-24). Particle size analysis of IBU-SLNs revealed that the SLN prepared with higher lipid concentration showed a larger particle size. To establish the effect of surfactant type on SLNs, we used two commonly used surfactants, Poloxamer 188 and Tween-80. We observed a lower particle size in Tween-80 based SLNs compared to Poloxamer SLN formulations. A possible explanation for this difference in particle size may be the effect of hydrocarbon chain length difference of the used surfactants (25). Thus, with regards to particle size, the concentration of lipid and type of emulsifier seem to be important process parameters. The results of $\%$ EE indicated that increasing the lipid concentration also increases encapsulation due to the presence of long chain fatty alcohols, which could lead to the less ordered solid lipid matrix and leave enough space to accommodate drug molecules (26). The high \% EE can also be attributed to the lipophilic nature of IBU.

Surface charge potential should play an important role in nanoparticle stability due to electrostatic repulsion. Since Poloxamer 188 and Tween- 80 are non-ionic surfactants, lipid cores may be responsible for the negative surface charge. The negative charge was likely caused by the slightly ionized fatty acids from glycerides. This may explain the changes in zeta potential produced by the amount of lipid. Patil et al reported that nanoparticle with zeta potential of $-43 \mathrm{mV}$ have the highest cellular uptake compared with other formulations with less negative and/or positive surface charge $(27,28)$. Nanoparticles show a high affinity for cellular membrane mainly due to electrostatic interactions $(29,30)$. These findings agree with results obtained in this study that IBU-SLNs with higher negative charge showed greater inhibition of cell viability. From in vitro drug release studies (Fig. 4), we observed that $80 \%$ of IBU drug was released in $24 \mathrm{~h}$, while the later phase lasted for $96 \mathrm{~h}$, which correlates with the physiological requirement for humans. For oral administration, both burst release and sustained drug release are of importance to ensure quick efficacy and prolonged drug presence. For our studies, the burst release occurred due to the presence of the free IBU in the external phase and on the surface of the SLNs. The lipophilic nature of the IBU could be the reason for sustained release of the drug from internal lipidic phase after initial burst release.

The cell viability assay showed that both free IBU and IBU-SLNs could significantly suppress cell viability in a dosedependent manner. The effect of these agents was evaluated by calculating the $\mathrm{IC}_{50}$ values of free IBU and IBU-SLNs. It was observed that IBU-SLNs $\mathrm{IC}_{50}$ concentrations exhibited approximately 10 -fold reductions in comparison to the $\mathrm{IC}_{50}$ of free form of IBU. Previous studies have been reported where drug loaded aspirin, curcumin, and sulforaphane SLNs have exhibited better cytotoxicity profile in comparison to the free drug on in vitro and in vivo models of pancreatic cancer $(2,4,5)$. In cell viability assay, DMSO and blank SLNs were used as control for free IBU and IBU-SLNs, respectively. We found 
that higher concentration of stearic acid (500 $\mu \mathrm{M}$ and above) in blank SLNs showed $\sim 30 \%$ reduction in cell viability, thus the cytotoxicity effect of low concentration of IBU-SLNs was confirmed to be because of IBU. The surfactants used as components of SLN, when tested alone at equivalent concentrations used in SLN, did not result in reduction of cell viability. Therefore, cytotoxic effects, induced by SLN formulations containing specific surfactants, did not appear to accrue from the surfactants used.

Single agent administration at low concentrations was demonstrated to be ineffective, hence the hypothesis that two or more chemopreventive agents when delivered at low concentrations together, may exhibit an additive or synergistic effect against the cancer cells was tested. This can be attributed to the multi-factorial nature of carcinogenesis wherein cancer initiates as a result of multiple cellular changes during a prolonged time period. Several in vitro and in vivo studies have shown that NSAIDs like aspirin and celecoxib have helped prevent the progression of pancreatic cancer (2,31-33). In our studies, the $\mathrm{IC}_{50}$ value of IBU-SLN was found to be $113.8 \mu \mathrm{M}$ and $122.6 \mu \mathrm{M}$ in Panc- 1 and MIA PACa- 2 cells, respectively. However, studies of ineffective and low dose of IBU-SLN $(62.5 \mu \mathrm{M})$ combined with SFN $(5 \mu \mathrm{M})$ exhibited a synergistic effect against the pancreatic cancer cells, proving to be more efficacious at lower concentrations, reducing the concentration of IBU-SLN by 4 times compared to free IBU $(250 \mu \mathrm{M})$ in combination with SFN. Therefore, using a multi-disciplinary approach, this study investigated the synergistic effects of SLN combinations of chemopreventive agents, namely, IBU-SLN in combination with free SFN.

From these results, we believe that chemoprevention is an effective way to prevent pancreatic cancer especially since the disease cannot be diagnosed at an early stage. Using a multi-disciplinary approach, this study investigated optimized formulation parameters for IBU-SLNs and the synergistic effects of a combination of IBU-SLN with free SFN. We demonstrated that this IBU-SLN combination with SFN showed a synergistic inhibition of cell viability in human pancreatic cancer cells. However, further in vivo studies have to be conducted to test the efficacy of IBU-SLN alone and in combination with SFN. Additionally, to assess the longterm benefits of this regimen, a toxicity safety study would be necessary. In conclusion, the preliminary results obtained from formulation studies and cell based assays clearly demonstrate the translational scope of developing low dose IBU encapsulated SLN formulations to prevent pancreatic cancer.

\section{References}

1. Siegel R, Ma J, Zou Z and Jemal A: Cancer statistics, 2014. CA Cancer J Clin 64: 9-29, 2014.

2. Grandhi BK, Thakkar A, Wang J and Prabhu S: A novel combinatorial nanotechnology-based oral chemopreventive regimen demonstrates significant suppression of pancreatic cancer neoplastic lesions. Cancer Prev Res 6: 1015-1025, 2013.

3. Chaudhary A, Sutaria D, Huang Y, Wang J and Prabhu S: Chemoprevention of colon cancer in a rat carcinogenesis model using a novel nanotechnology-based combined treatment system. Cancer Prev Res 4: 1655-1664, 2011.

4. Thakkar A, Sutaria D, Grandhi BK, Wang J and Prabhu S: The molecular mechanism of action of aspirin, curcumin and sulforaphane combinations in the chemoprevention of pancreatic cancer. Oncol Rep 29: 1671-1677, 2013.
5. Sutaria D, Grandhi BK, Thakkar A, Wang J and Prabhu S: Chemoprevention of pancreatic cancer using solid-lipid nanoparticulate delivery of a novel aspirin, curcumin and sulforaphane drug combination regimen. Int J Oncol 41: 2260-2268, 2012.

6. Palayoor ST, Bump EA, Calderwood SK, Bartol S and Coleman $\mathrm{CN}$ : Combined antitumor effect of radiation and ibuprofen in human prostate carcinoma cells. Clin Cancer Res 4: 763-771, 1998

7. Yao M, Zhou W, Sangha S, et al: Effects of nonselective cyclooxygenase inhibition with low-dose ibuprofen on tumor growth, angiogenesis, metastasis, and survival in a mouse model of colorectal cancer. Clin Cancer Res 11: 1618-1628, 2005.

8. Bonelli P, Tuccillo FM, Federico A, et al: Ibuprofen delivered by poly(lactic-co-glycolic acid) (PLGA) nanoparticles to human gastric cancer cells exerts antiproliferative activity at very low concentrations. Int J Nanomed 7: 5683-5691, 2012.

9. Lanas A: A review of the gastrointestinal safety data - a gastroenterologist's perspective. Rheumatology 49 (Suppl 2): ii3-ii10, 2010.

10. Mallen SR, Essex MN and Zhang R: Gastrointestinal tolerability of NSAIDs in elderly patients: a pooled analysis of 21 randomized clinical trials with celecoxib and nonselective NSAIDs. Curr Med Res Opin 27: 1359-1366, 2011.

11. Brigger I, Dubernet C and Couvreur P: Nanoparticles in cancer therapy and diagnosis. Adv Drug Deliv Rev 54: 631-651, 2002.

12. Potta SG, Minemi S, Nukala RK, et al: Preparation and characterization of ibuprofen solid lipid nanoparticles with enhanced solubility. J Microencapsul 28: 74-81, 2011.

13. Kokawa A, Kondo H, Gotoda T, et al: Increased expression of cyclooxygenase-2 in human pancreatic neoplasms and potential for chemoprevention by cyclooxygenase inhibitors. Cancer 91: 333-338, 2001.

14. O'Driscoll CM: Lipid-based formulations for intestinal lymphatic delivery. Eur J Pharm Sci 15: 405-415, 2002.

15. Mehnert W and Mäder K: Solid lipid nanoparticles: production, characterization and applications. Adv Drug Deliv Rev 47: 165-196, 2001.

16. Matusheski NV, Juvik JA and Jeffery EH: Heating decreases epithiospecifier protein activity and increases sulforaphane formation in broccoli. Phytochemistry 65: 1273-1281, 2004.

17. Fahey JW, Haristoy X, Dolan PM, et al: Sulforaphane inhibits extracellular, intracellular, and antibiotic-resistant strains of Helicobacter pylori and prevents benzo[a]pyrene-induced stomach tumors. Proc Natl Acad Sci USA 99: 7610-7615, 2002.

18. Kuroiwa Y, Nishikawa A, Kitamura Y, et al: Protective effects of benzyl isothiocyanate and sulforaphane but not resveratrol against initiation of pancreatic carcinogenesis in hamsters. Cancer Lett 241: 275-280, 2006.

19. Chung FL, Conaway CC, Rao CV and Reddy BS: Chemoprevention of colonic aberrant crypt foci in Fischer rats by sulforaphane and phenethyl isothiocyanate. Carcinogenesis 21: 2287-2291, 2000.

20. Kallifatidis G, Rausch V, Baumann B, et al: Sulforaphane targets pancreatic tumour-initiating cells by NF-kappaB-induced antiapoptotic signalling. Gut 58: 949-963, 2009.

21. Sze A, Erickson D, Ren L and Li D: Zeta-potential measurement using the Smoluchowski equation and the slope of the currenttime relationship in electroosmotic flow. J Colloid Interface Sci 261: 402-410, 2003.

22. Petersen S, Steiniger F, Fischer D, Fahr A and Bunjes H: The physical state of lipid nanoparticles influences their effect on in vitro cell viability. Eur J Pharm Biopharm 79: 150-161, 2011.

23. Schöler N, Hahn H, Müller RH and Liesenfeld O: Effect of lipid matrix and size of solid lipid nanoparticles (SLN) on the viability and cytokine production of macrophages. Int J Pharm 231: 167-176, 2002.

24. Schöler N, Olbrich C, Tabatt K, Müller RH, Hahn H and Liesenfeld O: Surfactant, but not the size of solid lipid nanoparticles (SLN) influences viability and cytokine production of macrophages. Int J Pharm 221: 57-67, 2001.

25. Arnarson T and Elworthy PH: Effects of structural variations on non-ionic surfactants on micellar properties and solubilization: surfactants containing very long hydrocarbon chains. J Pharm Pharmacol 33: 141-144, 1981.

26. Sanna V, Caria G and Mariani A: Effect of lipid nanoparticles containing fatty alcohols having different chain length on the ex vivo skin permeability of Econazole nitrate. Powder Technol 201: 32-36, 2010 
27. Patil S, Sandberg A, Heckert E, Self W and Seal S: Protein adsorption and cellular uptake of cerium oxide nanoparticles as a function of zeta potential. Biomaterials 28: 4600-4607, 2007.

28. Bernfield M, Götte M, Park PW, et al: Functions of cell surface heparan sulfate proteoglycans. Annu Rev Biochem 68: 729-777, 1999.

29. Win KY and Feng SS: Effects of particle size and surface coating on cellular uptake of polymeric nanoparticles for oral delivery of anticancer drugs. Biomaterials 26: 2713-2722, 2005.

30. Cheng H, Zhu JL, Zeng X, Jing Y, Zhang XZ and Zhuo RX: Targeted gene delivery mediated by folate-polyethylenimineblock-poly(ethylene glycol) with receptor selectivity. Bioconjug Chem 20: 481-487, 2009.
31. Al-Wadei HA, Al-Wadei MH, Ullah MF and Schuller HM: Celecoxib and GABA cooperatively prevent the progression of pancreatic cancer in vitro and in xenograft models of stress-free and stress-exposed mice. PLoS One 7: e43376, 2012.

32. Mukherjee P,Basu GD, Tinder TL, et al: Progression of pancreatic adenocarcinoma is significantly impeded with a combination of vaccine and COX-2 inhibition. J Immunol 182: 216-224, 2009.

33. Streicher SA, Yu H, Lu L, Kidd MS and Risch HA: Case-control study of aspirin use and risk of pancreatic cancer. Cancer Epidemiol Biomarkers Prev 23: 1254-1263, 2014. 